\title{
Range-Ambiguous Clutter Suppression with Pulse-Diverse Waveforms
}

\author{
Dan P. Scholnik \\ Radar Division, Naval Research Laboratory, Washington, DC
}

\begin{abstract}
Conventional pulse-Doppler waveforms are the standard for radar detection in clutter due to their inherent robustness, especially to extended-range ambiguous clutter. The attendant drawbacks include coherent ambiguities and blind zones in range and/or Doppler, which require multiple coherent precessing intervals to resolve. Pulse-diverse waveforms, using techniques such as phase coding and nonuniform time offsets, can mitigate these drawbacks but at the cost of a significant reduction in clutter suppression using matched filtering. It is shown here that receive filtering optimized on a per-interval or per-range/Doppler-cell basis can provide these waveforms with clutter suppression over multiple range intervals, at the cost of significantly increased computation. Several examples illustrate the tradeoffs between different types of pulse diversity.
\end{abstract}

\section{INTRODUCTION}

A single coherent processing interval (CPI) in traditional pulseDoppler radar consists of repeatedly transmitting and receiving a common pulse with a fixed pulse repetition interval (PRI). Such waveforms have advantages both in Doppler robustness and in processing requirements. The former is due to the inherent insensitivity to fixed pulse distortions and the ability to suppress Doppler sidelobes over the entire ambiguous Doppler interval. The latter is due to the fast-time/slow-time separability of the receive processing into fast convolution for pulse compression and FFT-based Doppler processing. The ambiguity in range and/or Doppler that results from these waveforms is a two-edged sword: by collapsing all range intervals together it simplifies processing and allows cancellation of extendedrange ambiguous clutter, but resolving these ambiguities and filling in the blind zones requires multiple CPIs using different PRIs.

Pulse-diverse waveforms, defined here generally as those in which each pulse differs from the next by some combination of phase, phase code, time offset, and frequency shift, feature suppressed coherent range ambiguities. Those including nonuniform time offsets can suppress Doppler ambiguities as well. The drawback is that with matched filtering the ambiguity is smeared across range and Doppler, and for practical waveforms remains at too high a level to suppress clutter. Thus these waveforms have received far less attention, and it was generally believed that they could not be used in the presence of multiple-time-around clutter [1]. However, [2] and [3] have showed ambiguous clutter cancellation for "coherent on receive" waveforms, so-called because the starting phase of each transmit pulse is random.

In fact, clutter cancellation is possible for a much wider range of pulse-diverse waveforms, as will be shown in the sequel. Such an approach is very tempting, as it promises a reduction in dwell time now that ambiguity resolution is not needed. As expected, there are drawbacks. In general, unique filters are needed for each range/Doppler bin, greatly increasing the required processing. A great deal of robustness to unknown clutter distributions is lost, although these can be mitigated using an iterative approach akin to adaptive pulse compression and its descendants [4], [5]. There are still constraints on the total range-Doppler area of clutter that can be canceled. Finally, the robustness to uncorrected pulse errors is lost, and thus the actual transmitted pulses must be known precisely.

\section{FRAMEWORK}

A brief overview of the radar linear-processing problem and its restriction to narrowband signals is presented to define a generalized cross-ambiguity function. A simple processing model is then employed to explicitly show the dependence on digital receive-filter coefficients, which will be optimized in Section III.

\section{A. Generalized Cross-Ambiguity Function}

Given transmit waveform $x(t)$, let $a x^{\prime}\left(t ; R^{\prime}, v^{\prime}\right)$ be the scaled, delayed, and Doppler-shifted return from a point target at range $R^{\prime}$ with velocity $v^{\prime}$. The total received waveform is a sum of all such returns:

$$
y(t)=\iint x^{\prime}\left(t ; R^{\prime}, v^{\prime}\right) a\left(R^{\prime}, v^{\prime}\right) d R^{\prime} d v^{\prime}
$$

where $a$ is the target distribution in range and velocity. The general linear estimate of the amplitude of a potential target return from a given range $R$ and velocity $v$ is obtained via the inner product

$$
M(R, v)=\int h^{*}(t ; R, v) y(t) d t
$$

where $h$ is a filter parametrized on $(R, v)$ and $M$ is the resulting range-Doppler map. Expanding $y$ and rearranging yields

$$
M(R, v)=\iint \chi_{h x}\left(R, v, R^{\prime}, v^{\prime}\right) a\left(R^{\prime}, v^{\prime}\right) d R^{\prime} d v^{\prime}
$$

where

$$
\chi_{h x}\left(R, v, R^{\prime}, v^{\prime}\right) \triangleq \int h^{*}(t ; R, v) x^{\prime}\left(t ; R^{\prime}, v^{\prime}\right) d t
$$

is the generalized cross-ambiguity function (CAF) of waveform $x$ and filter $h$. Notably, both $M$ and the CAF are linear in the filter $h$, and thus are amenable to convex optimization when the transmit waveform is chosen a priori.

\section{B. Narrowband Assumption}

The results of (2) and (3) are quite general in that they assume no particular model for $x^{\prime}$ and impose no structure on $h$. In particular, both wideband and narrowband models can be used, and effects such as transmit blanking can be directly included. Of primary interest here is the narrowband assumption combined with transmit blanking. Under the narrowband assumption we have

$$
x^{\prime}\left(t ; R^{\prime}, v^{\prime}\right)=x\left(t-\tau_{R^{\prime}}\right) e^{j 2 \pi v_{v^{\prime}}\left(t-\tau_{R^{\prime}}\right)}
$$

where $\tau_{R^{\prime}}=2 R^{\prime} / c$ is the range delay and $v_{v^{\prime}}=-2 f_{0} v^{\prime} / c$ is the Doppler frequency shift that results from velocity $v$ at an RF of $f_{0}$. Now the ambiguity function can be written

$$
\chi_{h x}\left(R, v ; R^{\prime}, v^{\prime}\right)=\int h^{*}(t ; R, v) x\left(t-\tau_{R^{\prime}}\right) e^{j 2 \pi v_{v^{\prime}}\left(t-\tau_{R^{\prime}}\right)} d t
$$

which for each $(R, v)$ pair is the conventional narrowband crossambiguity function of $h$ and $x$.

The narrowband representation also lends itself to a convenient statistical characterization of the range-Doppler map more appropriate for modeling clutter. Let the response $b\left(R^{\prime} ; t\right)$ at each range $R^{\prime}$ be a 
wide-sense stationary random process uncorrelated from other ranges, with autocorrelation $r_{b}\left(R^{\prime} ; t^{\prime}\right)=E\left\{b\left(R^{\prime} ; t\right) b^{*}\left(R^{\prime} ; t-t^{\prime}\right)\right\}$. Then the output becomes

$$
y(t)=\int x\left(t-\tau_{R^{\prime}}\right) b\left(t ; R^{\prime}\right) d R^{\prime}
$$

and we can characterize the expected power in the range-Doppler map as

$$
E\left\{|M(R, v)|^{2}\right\}=\iint\left|\chi_{h x}\left(R, v ; R^{\prime}, v^{\prime}\right)\right|^{2} S_{b}\left(R^{\prime} ; v_{v^{\prime}}\right) d R^{\prime} d v^{\prime}
$$

where spectral density $S_{b}$ is the Fourier transform (on lag variable $\left.t^{\prime}\right)$ of $r_{b}$. Here we see that $|M(R, v)|^{2}$ serves as a biased estimate of $S_{b}$, with the CAF serving as a shift-varying point-spread function.

\section{Discrete-Time Representations}

To this point the waveform $x$ and filter response $h$ have been represented in continuous time, but in modern systems the bulk of the design control lies in the digital domain. Here we explicitly factor the discrete-time and continuous-time components on both transmit and receive to expose the filter coefficients that will be optimized in the following section. In addition, under relatively mild conditions it is argued that it is sufficient to design the digital receive filter based only on the digital transmit waveform independent of the associated analog filters.

We will represent all analog filters in the receiver as the (known, fixed) LTI response $g(t)$, the output of which is the convolution

$$
(g * y)\left(t^{\prime}\right) \triangleq \int g\left(t^{\prime}-t\right) y(t) d t .
$$

This signal is sampled at a rate $1 / T_{S}$, and an inner product is taken with the discrete-time sequence $\beta_{n}(R, v)$ to yield the range-Doppler map:

$$
\begin{aligned}
M(R, v) & =\sum_{n} \beta_{n}^{*}(R, v)(g * y)\left(n T_{s}\right) \\
& =\int\left(\sum_{n} \beta_{n}^{*}(R, v) g\left(n T_{s}-t\right)\right) y(t) d t .
\end{aligned}
$$

Comparing this to (1) yields a mixed discrete-time/continuous-time convolution representation of $h$ :

$$
h(t ; R, v)=\sum_{n} \beta_{n}(R, v) g^{\dagger}\left(t-n T_{s}\right)
$$

where $g^{\dagger}(t) \triangleq g^{*}(-t)$. The filter coefficients $\left\{\beta_{n}\right\}$ represent the variables to be optimized for each $(R, v)$.

A similar decomposition will prove convenient for waveforms that can be generated using linear pulse modulation. This directly includes two common constant-envelope modulations used for phase-coded waveforms, namely minimum-shift keying (MSK) and instantaneous phase switching, and indirectly includes any bandlimited signal under Nyquist sampling. Here we can represent the transmitted waveform $x(t)$ as the mixed convolution

$$
x(t)=\sum_{n} \alpha_{n} p\left(t-n T_{c}\right) .
$$

Substituting into (4) yields

$$
x^{\prime}\left(t ; R^{\prime}, v^{\prime}\right)=\sum_{n} \alpha_{n} p\left(t-\tau_{R^{\prime}}-n T_{c}\right) e^{j 2 \pi v_{v^{\prime}}\left(t-\tau_{R^{\prime}}\right)} .
$$

If we further assume that the support of $p$ is concentrated close enough to the time origin that the exponential factor is approximately constant over its duration, then we can simplify this as

$$
x^{\prime}\left(t ; R^{\prime}, v^{\prime}\right)=\sum_{n} \alpha_{n} e^{j 2 \pi v_{v^{\prime}} n T_{c}} p\left(t-\tau_{R^{\prime}}-n T_{c}\right)
$$

which is the mixed convolution of $p$ with a Doppler-shifted version of the original transmit sequence. Effectively we have assumed that the pulse shaping is invariant to Doppler shifts of interest.

After substituting (6) and (7) into (3), rearranging, and identifying a convolution, we can write the narrowband CAF as

$\chi_{h x}\left(R, v, R^{\prime}, v^{\prime}\right)=\sum_{k} \beta_{k}^{*}(R, v) \sum_{n} \alpha_{n} e^{j 2 \pi v_{v^{\prime}} n T_{c}}(g * p)\left(k T_{s}-n T_{c}-\tau_{R^{\prime}}\right)$.

Defining the sequence

$$
\gamma_{k}\left(R^{\prime}, v^{\prime}\right) \triangleq \sum_{n} \alpha_{n} e^{j 2 \pi v_{v^{\prime}} n T_{c}}(g * p)\left(k T_{s}-n T_{c}-\tau_{R^{\prime}}\right)
$$

which results from receiving and sampling $x^{\prime}\left(t ; R^{\prime}, v^{\prime}\right)$, reveals that the CAF is the inner product of $\beta(R, c)$ and $\gamma\left(R^{\prime}, v^{\prime}\right)$. Vectorizing these as $\boldsymbol{\beta}$ and $\boldsymbol{\gamma}$ yields

$$
\chi_{h x}\left(R, v ; R^{\prime}, v^{\prime}\right)=\boldsymbol{\beta}^{H}(R, v) \boldsymbol{\gamma}\left(R^{\prime}, v^{\prime}\right)
$$

a form which will be useful in the next section.

Returning to (8), it will be convenient to assume that $T_{S}=T_{c}$. Although this seems restrictive, either or both of $\alpha$ or $\beta$ can be zero-interpolated such that $T_{S}$ is merely the GCD of the true sample spacings. Further making the change of variable $l=k-n$ and defining the discrete-time CAF as

$$
D_{\beta \alpha}\left(R, v ; l, v^{\prime} T_{S}\right) \triangleq \sum_{k} \beta_{k}^{*}(R, v) \alpha_{k-l} e^{j 2 \pi v_{v^{\prime} T_{S}}(k-l)}
$$

yields the expression

$$
\chi_{h x}\left(R, v, R^{\prime}, v^{\prime}\right)=\sum_{l} D_{\beta \alpha}\left(R, v ; l, v^{\prime} T_{S}\right)(g * p)\left(l T_{S}-\tau_{R^{\prime}}\right) .
$$

Thus we can represent the continuous-time CAF as a delayed mixed convolution of the discrete-time $\mathrm{CAF}$ and the combined analog filtering in the system.

The preceding derivation suggests it is often sufficient to design the discrete-time CAF in isolation, reducing design computation and bypassing the need to know $p$ and $g$. In effect, $D_{\beta \alpha}$ provides the coarse profile of the full CAF, while the filters $g$ and $p$ merely provide interpolation/smoothing in range. Geometries where this is relatively harmless include isolated mainlobes (the edges of which are spread in range as would be expected due to the system bandwidth) and extended-range low-sidelobe regions. (The edges of the latter might also suffer some spreading, although this could be accommodated by increasing the stopband width accordingly.) This approximation is less useful for narrow notches in range, which would tend to be filled in if not sufficiently widened.

\section{Transmit Blanking}

To represent transmit blanking, let $\mathcal{X}$ be the support of the transmitted waveform, and $\overline{\mathcal{X}}$ its complement. Then the blanked narrowband return can be written

$$
x_{b}^{\prime}\left(t ; R^{\prime}, v^{\prime}\right)=1_{\overline{\mathcal{X}}}(t) x\left(t-\tau_{R^{\prime}}\right) e^{j 2 \pi v_{v^{\prime}}\left(t-\tau_{R^{\prime}}\right)}
$$

where $1_{\overline{\mathcal{X}}}$ is the indicator function on $\overline{\mathcal{X}}$. The blanked CAF is

$$
\chi_{h x_{b}}\left(R, v ; R^{\prime}, v^{\prime}\right)=\int 1_{\overline{\mathcal{X}}}(t) h^{*}(t ; R, v) x\left(t-\tau_{R^{\prime}}\right) e^{j 2 \pi v_{v^{\prime}}\left(t-\tau_{R^{\prime}}\right)} d t
$$

revealing that the blanking can be incorporated directly into the definition of the filter as regions of enforced zero value. Typically this is done by reducing the support of the digital filter $\beta$, which represents dropped samples during the blanking interval. 


\section{OPTIMIZING THE CAF}

While it is common in conventional range-Doppler processing to derive all of the filters from a single prototype $h_{0}(t)$ as

$$
h(t ; R, v)=h_{0}\left(t-\tau_{R}\right) e^{j 2 \pi v_{v}\left(t-\tau_{R}\right)}
$$

to gain considerable computational efficiency, in general unique filters for each range/Doppler pair $(R, v)$ are needed when processing pulsediverse waveforms. Fundamentally this is because such waveforms support much smaller low-sidelobe regions in the CAF, and thus a single filter can't provide the required suppression over all shifts in range and Doppler. In addition, transmit blanking destroys the shiftinvariance of the returned waveform and thus partially blanked returns require custom filters (if they are to be processed at all). Some of the efficiency of conventional processing can be retained at the expense of reduced flexibility and performance; for example, [6] represents a special case of the present work where shift-invariance is maintained in range and the filter support is restricted to that of the matched filter. This allows the use of fast convolution but restricts clutter suppression to relative range offsets rather than absolute ranges.

Many constrained and unconstrained optimization approaches can be used to find the receive filters $h$ that optimize the CAF in some sense. Two are considered here: a constrained approach which offers finer control and is suited primarily for offline processing, and a more efficient unconstrained approach suitable for both offline and adaptive processing.

\section{A. Second-Order Cone Programming Approach}

The first optimization approach uses second-order cone programming (SOCP), a structured form of convex optimization that minimizes a linear objective subject to linear and convex-quadratic constraints [7]. SOCP has found wide use in filter design due to its combination of flexibility and efficiency. The advantage of constrained optimization here is that it's possible to set specific bounds on certain error terms of interest while minimizing another.

As an example, for each $(R, v)$ of interest, consider the SOCP design

$$
\begin{aligned}
\underset{\left\{\beta_{n}(R, v)\right\}, \delta}{\min } & \delta \geq 0 \\
\text { s.t. } & \|\beta(\cdot ; R, v)\|^{2}\|\gamma(\cdot ; R, v)\|^{2} \leq \delta^{2} \\
& \chi_{h x}(R, v ; R, v)=1 \\
& \frac{1}{\iint_{\mathcal{S}} d R^{\prime} d v^{\prime}} \iint_{\mathcal{S}}\left|\chi_{h x}\left(R, v ; R^{\prime}, v^{\prime}\right)\right|^{2} d R^{\prime} d v^{\prime} \leq 10^{-\frac{80}{10}}
\end{aligned}
$$

Converting a specification such as this into canonical SOCP form is addressed in [7]-[9]. Here $\delta$ is an auxiliary variable used for the linear objective (12a), which combined with quadratic constraint (12b) minimizes the noise gain of the filter $\beta$. The mismatch penalty of $\beta$ with respect to received and sampled waveform $\gamma$ in white noise (added at the sampler) is given as $\frac{\|\boldsymbol{\beta}\|^{2}\|\boldsymbol{\gamma}\|^{2}}{\left|\boldsymbol{\beta}^{H} \boldsymbol{\gamma}\right|^{2}}$. By the Cauchy-Schwarz inequality, this fraction is bounded below by unity. Linear constraint (12c), which ensures unity gain for the desired target, also sets the denominator of the mismatch penalty to unity thus making (12b) an upper bound on the mismatch penalty. Finally, (12d) sets the meansquare clutter suppression on region $\mathcal{S}$ to $80 \mathrm{~dB}$. Alternatively, a fixed upper bound on the mismatch penalty could be used while minimizing the mean-square clutter gain. Peak rather than meansquare clutter suppression can be constrained by replacing the single quadratic constraint (12d) with the collection of constraints

$$
\left|\chi_{h x}\left(R, v ; R_{k}^{\prime}, v_{k}^{\prime}\right)\right|^{2} \leq 10^{-\frac{80}{10}} ; \quad\left(R_{k}^{\prime}, v_{k}^{\prime}\right) \in \mathcal{S}
$$

on a dense set of points $\left\{\left(R_{k}^{\prime}, v_{k}^{\prime}\right)\right\}$ over the sidelobe region $\mathcal{S}$.

\section{B. MVDR Approach}

Although SOCP is a very efficient optimization method, constrained optimization by its nature is more complicated to solve than unconstrained. A more important drawback here is that the solutions for different $(R, v)$ are independent; in general there is no way to simultaneously solve for multiple filters. An alternative approach based on the well-known minimum-variance distortionless response (MVDR) beamforming problem provides both a quicker solution and the ability to simultaneously solve for all filters that share the same support.

Fundamentally, the MVDR problem of interest here is:

$$
\begin{array}{cl}
\min _{\left\{\beta_{n}(R, v)\right\}} & E\left\{|M(R, v)|^{2}\right\}+\sigma^{2}\|\boldsymbol{\beta}\|^{2} \\
\text { s.t. } & \chi_{h x}(R, v ; R, v)=1
\end{array}
$$

minimizing the total power in each range-Doppler map cell (including due to white noise at the sampler with variance $\sigma^{2}$ ) while ensuring unity gain for a target actually at that range and Doppler. In standard MVDR form this is

$$
\begin{array}{ll}
\min _{\boldsymbol{\beta}(R, v)} & \boldsymbol{\beta}^{H} \mathbf{Q} \boldsymbol{\beta} \\
\text { s.t. } & \boldsymbol{\beta}^{H} \boldsymbol{\gamma}(R, v)=1
\end{array}
$$

where symmetric, positive-definite matrix $\mathbf{Q}$ is

$$
\mathbf{Q} \triangleq \iint \boldsymbol{\gamma}\left(R^{\prime}, v^{\prime}\right) \boldsymbol{\gamma}^{H}\left(R^{\prime}, v^{\prime}\right) S_{b}\left(R^{\prime} ; v_{v^{\prime}}\right) d R^{\prime} d v^{\prime}+\sigma^{2} \mathbf{I}
$$

The first term of $\mathbf{Q}$ results from substituting (9) into (5). The solution to the MVDR problem is then the well-known result

$$
\boldsymbol{\beta}_{\text {opt }}(R, v)=\frac{\mathbf{Q}^{-1} \boldsymbol{\gamma}(R, v)}{\boldsymbol{\gamma}^{H}(R, v) \mathbf{Q}^{-1} \boldsymbol{\gamma}(R, v)} .
$$

Of particular interest is that $\mathbf{Q}$ is the same for all $(R, v)$ pairs as long as the underlying filter support is the same. Since the bulk of the computation in evaluating (15) once is the inversion/decomposition of $\mathbf{Q}$, the marginal cost of evaluating (15) for additional steering vectors is minimal. Thus it is a highly efficient method to compute multiple filters at once.

\section{EXAMPLES}

In this section we will consider three different types of pulsediverse waveforms, and explore the properties of each through an example design.

\section{A. Phase-Coded Pulses w/ Fixed PRI}

In the first example the transmit waveform consists of 20 randomly biphase-coded pulses of 10 chips each with a fixed PRI and a $10 \%$ duty cycle. The receive filter was optimized using SOCP according to (12), substituting the discrete-time CAF to sidestep the need to model the analog filtering. The clutter region $\mathcal{S}$ extends over the first 7 PRIs in range and 0.001 PRF in Doppler. The support of filter $\beta$ consists of all non-blanked samples corresponding to returns from $\mathcal{S}$, which in this case was about 2400 samples total. The optimized CAF for a representative filter at a normalized range of 3.4 PRI and Doppler of $0.2 \mathrm{PRF}$ is shown in Fig. 1. The CAF sidelobes are concentrated according to the duty cycle; the RMS level of the significant sidelobes outside the clutter notch is approximately $1 / 200$ or $23 \mathrm{~dB}$, consistent with the transmit-waveform time-bandwidth product of 200. Although the waveform is unambiguous in range, the fixed PRF means that its CAF is ambiguous in Doppler and it has PRI-spaced blind zones in range. 


\section{B. Phase-Coded Pulses w/ Nonuniform PRI}

In the second example, the transmit waveform again consists of 20 randomly phase-coded pulses of 10 chips each with a $10 \%$ duty cycle. In this case, though, the pulse start times were randomized across 20 uniformly spaced time slots within the nominal PRI. The clutter region $\mathcal{S}$ was retained, and the same nominal filter location was optimized as before using SOCP. The receive filter support was again all samples corresponding to unblanked returns from ranges in $\mathcal{S}$, with approximately the same total length.

Some notable advantages of randomizing the PRI are apparent from the resulting optimized CAF, shown in Fig. 2. The RMS sidelobe level is approximately $1 / 2000$ or $-33 \mathrm{~dB}$; this is about ten times lower than with a uniform PRI as the sidelobe energy is spread out over the duty cycle. Thus the effective time-bandwidth product is increased without increasing total transmit time. In addition to the lower sidelobe levels, the CAF no longer has coherent Doppler ambiguities (although the Doppler sidelobes on the zero-range axis are poor for this short waveform). Another advantage is not visible from this single snapshot - there are no blind ranges beyond the beginning of the first interval. The staggered blanking intervals mean that all ranges lose on average $10 \%$ of their returns, rather than $10 \%$ losing all of their returns. This energy loss (seen here as a slightly increased mismatch penalty) can be compensated for by lengthening the waveform accordingly.

\section{Random Start Phases (Coherent-On-Receive)}

An important special case of pulse diversity is the so-called coherent-on-receive waveform [1], which has a random starting phase applied to otherwise identical pulses transmitted at a fixed PRI. As will be shown, this waveform allows the factorization of fast-time and slow-time processing in a computationally efficient manner.

Define the coherent-on-receive waveform as

$$
x(t)=\sum_{k=0}^{K-1} c_{k} x_{0}(t-k T)
$$

where $x_{0}$ is the common prototype pulse, the $\left\{c_{k}\right\}$ are unit-magnitude complex numbers and $T$ is the PRI. Similarly, the receive filtering is assumed to have the form

$$
h(t ; R, v)=\sum_{m=0}^{M-1} d_{m}(R, v) h_{0}(t-m T ; R, v) .
$$

Substituting (16) and (17) into (11) with a change of variable yields

$$
\begin{array}{r}
\chi_{h x_{b}}\left(R, v ; R^{\prime}, v^{\prime}\right)=\sum_{k=0}^{K-1} \sum_{m=0}^{M-1} c_{k} d_{m}^{*}(R, v) e^{j 2 \pi v_{v^{\prime}}} k T / 1_{\overline{\mathcal{X}}}(t+m T) \times \\
h_{0}^{*}(t ; R, v) x_{0}\left(t-\tau_{R^{\prime}}-(k-m) T\right) e^{j 2 \pi v_{v^{\prime}}\left(t-\tau_{R^{\prime}}-(k-m) T\right)} d t .
\end{array}
$$

Assuming that for uniformity we will continue to blank the receiver even after all pulses have been transmitted has the effect of periodically extending the blanking region so that $\mathcal{X}=\mathcal{X}+T$. Now we can write (18) as

$$
\begin{aligned}
& \chi_{h x_{b}}\left(R, v ; R^{\prime}, v^{\prime}\right)= \\
& \sum_{k=0}^{K-1} \sum_{m=0}^{M-1} c_{k} d_{m}^{*}(R, v) e^{j 2 \pi v_{v^{\prime}} k T} \chi_{h x_{0 b}}\left(R, v ; R^{\prime}+(k-m) c T / 2, v^{\prime}\right)
\end{aligned}
$$

where $\chi_{h x_{0 b}}$ is the blanked CAF of prototype pulse $x_{0}$ and prototype filter $h_{0}$. Finally, the change of variable $\ell=m-k$ yields

$$
\chi_{h x_{b}}\left(R, v ; R^{\prime}, v^{\prime}\right)=\sum_{\ell=-K+1}^{M-1} D_{d c}\left(R, v ; \ell, v^{\prime} T\right) \chi_{h x_{0 b}}\left(R, v ; R^{\prime}-\ell c T / 2, v^{\prime}\right)
$$

where we recognize the discrete-time CAF from (10), here applied to the slow time sequences $c$ and $d$. Thus for each filter location $(R, v)$ the overall CAF in range interval $\ell$ is the product of the slowtime CAF at $\ell$ and the blanked prototype CAF shifted by $\ell c T / 2$. All fine range detail within each interval is determined by $\chi_{h x_{0 b}}$, while the interval-to-interval range response and in most cases all of the Doppler detail is determined by $D$. This lets us design the slow-time filtering independently of the individual pulses.

Although the general formulation allows the prototype filter to compensate somewhat for transmit blanking, in most cases the prototype filter will be a fixed pulse-compression filter of the form $h_{0}(t ; R, v)=h_{\mathrm{pc}}\left(t-\tau_{R}\right)$ and only the coefficients $\left\{d_{m}\right\}$ need to be computed for each $(R, v)$. Here the output (1) becomes

$$
M(R, v)=\sum_{m=0}^{M-1} d_{m}^{*}(R, v)\left(h_{\mathrm{pc}}^{\dagger} * y\right)\left(\tau_{R}+m T\right)
$$

showing the separation of the outer, slow-time sum from the inner, fast-time pulse compression. This represents considerable computational savings over the general case where fast-time and slow-time processing are not separable or shift-invariant.

1) Known Spectral Density Example: For the first coherent-onreceive design we assume that the spectral density $S_{b}$ is known for a given range and its integer-PRI offsets. Clutter with a Gaussianshaped spectrum of standard deviation 0.002 PRF occupies the first 8 range intervals, and a single target is in interval 4 with a Doppler of 0.11 PRF. The clutter-to-noise and post-pulse-compression signal-tonoise ratios are $85 \mathrm{~dB}$ and $5 \mathrm{~dB}$, respectively. The transmit waveform consists of 100 pulses, and 120 return PRIs are processed to fully cover 21 range intervals. The slow-time CAF for a given range cell was optimized across the 21 range intervals and across one PRF interval in Doppler using MVDR according to (14a), here substituting slow-time coefficient vector $\mathbf{d}$ for fast-time vector $\boldsymbol{\beta}$ and likewise replacing fast-time steering vector $\boldsymbol{\gamma}\left(R^{\prime}, v^{\prime}\right)$ with slow time steering vector $\boldsymbol{\xi}\left(l^{\prime}, v^{\prime}\right)$, with $\xi_{k}\left(l^{\prime}, v^{\prime}\right) \triangleq c_{k-l^{\prime}} e^{j 2 \pi v_{v^{\prime} T}\left(k-l^{\prime}\right)}$. (The fast-time CAF of the prototype pulse was assumed to contribute negligibly to the Doppler response.)

A sample return was simulated with the given target, clutter, and noise, and the optimized filters were applied to compute the rangeDoppler app. The optimized slow-time CAF for a representative filter and the computed map are shown in the top two plots of Fig. 3. As before, the CAF shows a deep clutter notch and a single peak at the filter center. The range-Doppler map shows a well-defined clutter ridge and the single isolated target as desired.

2) Iterative Example: When the clutter spectrum is not known, it is possible to use an iterative approach in the spirit of adaptive pulse compression [4], [5]. Here we start with the matched-filter rangeDoppler map, and use that as an estimate of the spectral density $S_{b}$ to compute a first set of optimized filters using the MVDR approach. These filters are then used to compute a new range-Doppler map, and the two steps are repeated until the map converges. The map produced by matched-filtering is shown at bottom-left in Fig. 3, and the map that results after 20 iterations is shown at bottom right. The entire map is initially obscured by clutter using the matched filter, but is nearly identical to the clairvoyant version after iterating.

\section{Conclusions}

The analysis presented here shows that in principle it is possible to combine thumbtack-type ambiguity functions with high clutter suppression when custom filter optimization is used. Omitted for space considerations, but certainly not for lack of importance, is consideration of hardware precision. In practice, uncalibrated transmit errors are expected to be the limiting factor on clutter suppression. 


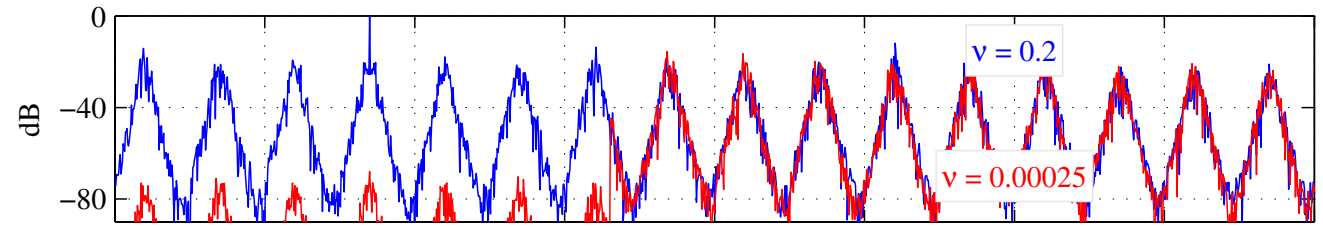

$$
\mathrm{R}=3.4
$$
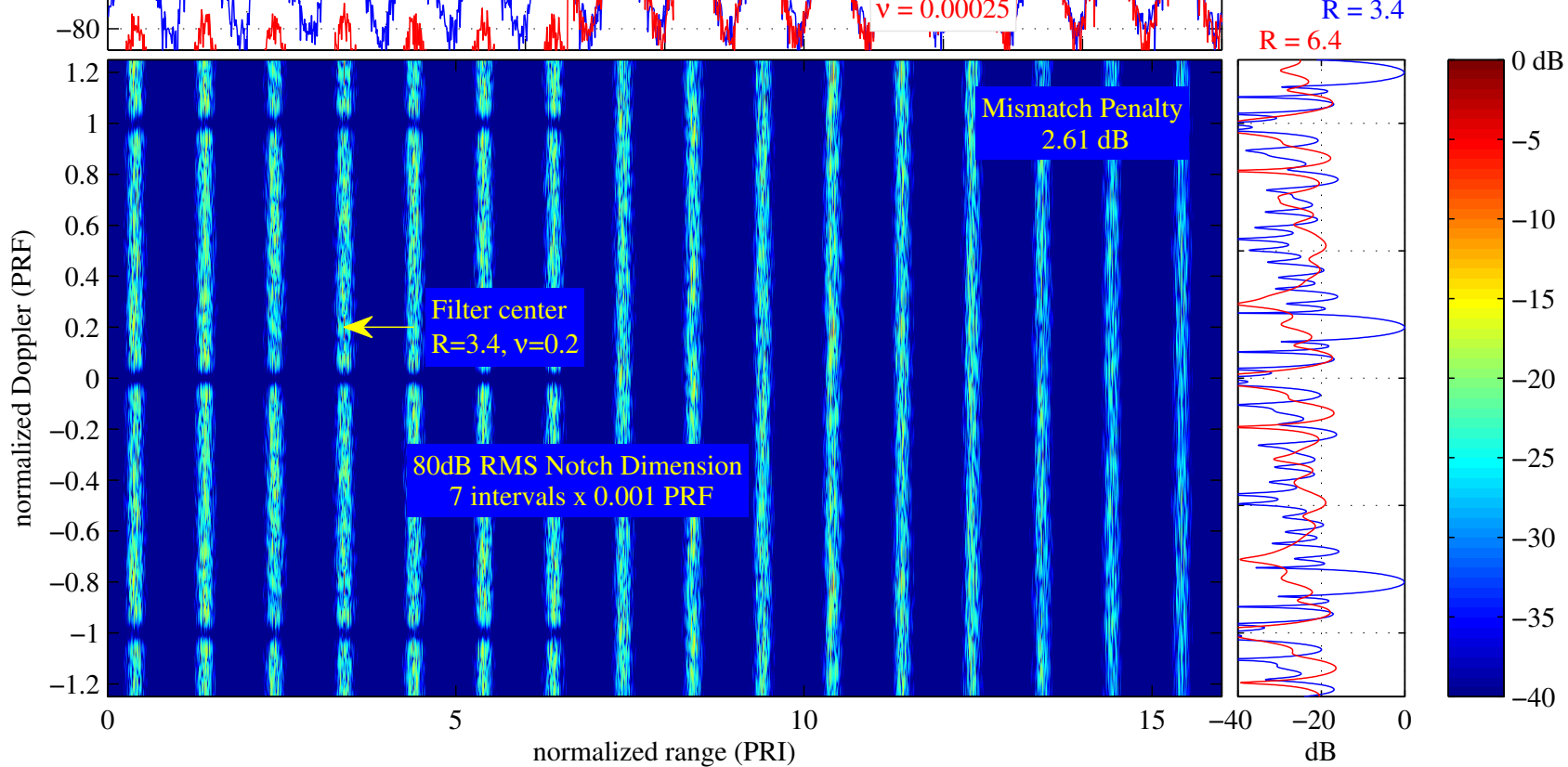

normalized range (PRI)

$\begin{array}{lll}15 & -40 & -20 \\ & & d B\end{array}$

Fig. 1. The optimized cross-ambiguity function for the first example, representing the filter centered at a normalized range of 3.4 and a normalized Doppler of 0.2. A clutter notch was imposed over the first 7 range intervals. Range cuts through the target Doppler and through the clutter notch are shown above, while Doppler cuts through the target range and a representative non-target range are shown to the right.

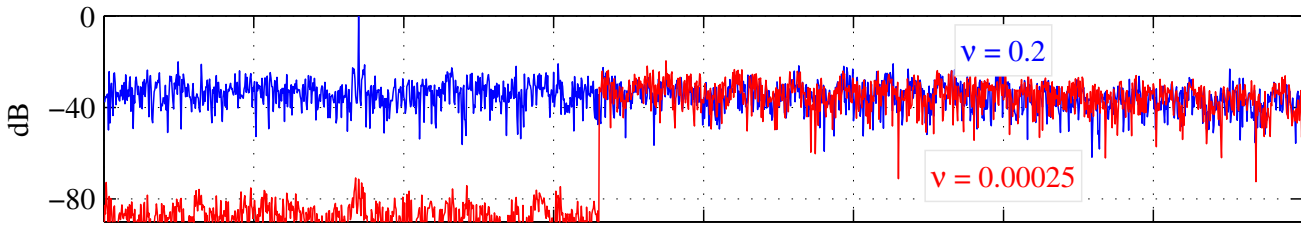

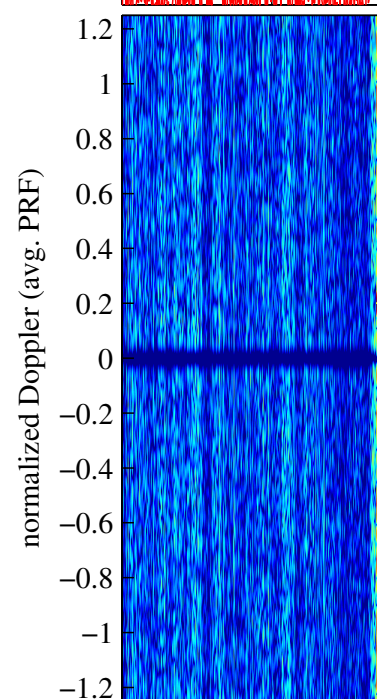

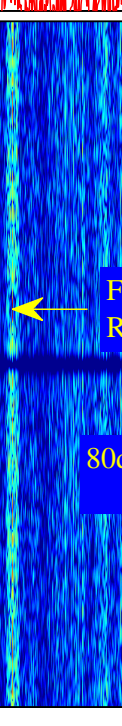
normalized range (avg. PRI)
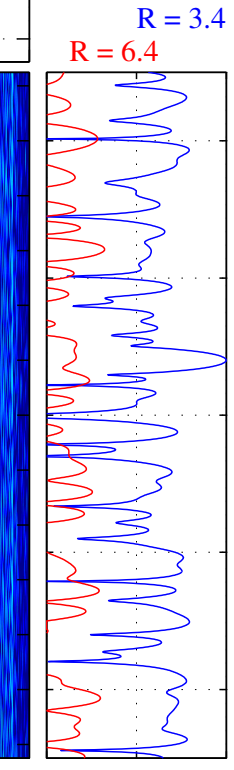

$\mathrm{R}=3.4$

15

$-40$

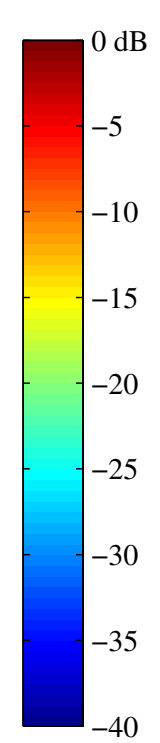

$\mathrm{dB}$

Fig. 2. The optimized cross-ambiguity function for the second example, representing filter location centered at a normalized range of 3.4 and a normalized Doppler of 0.2. A clutter notch was imposed over the first 7 range intervals. Range cuts through the target Doppler and through the clutter notch are shown above, while Doppler cuts through the target range and a representative non-target range are shown to the right. 

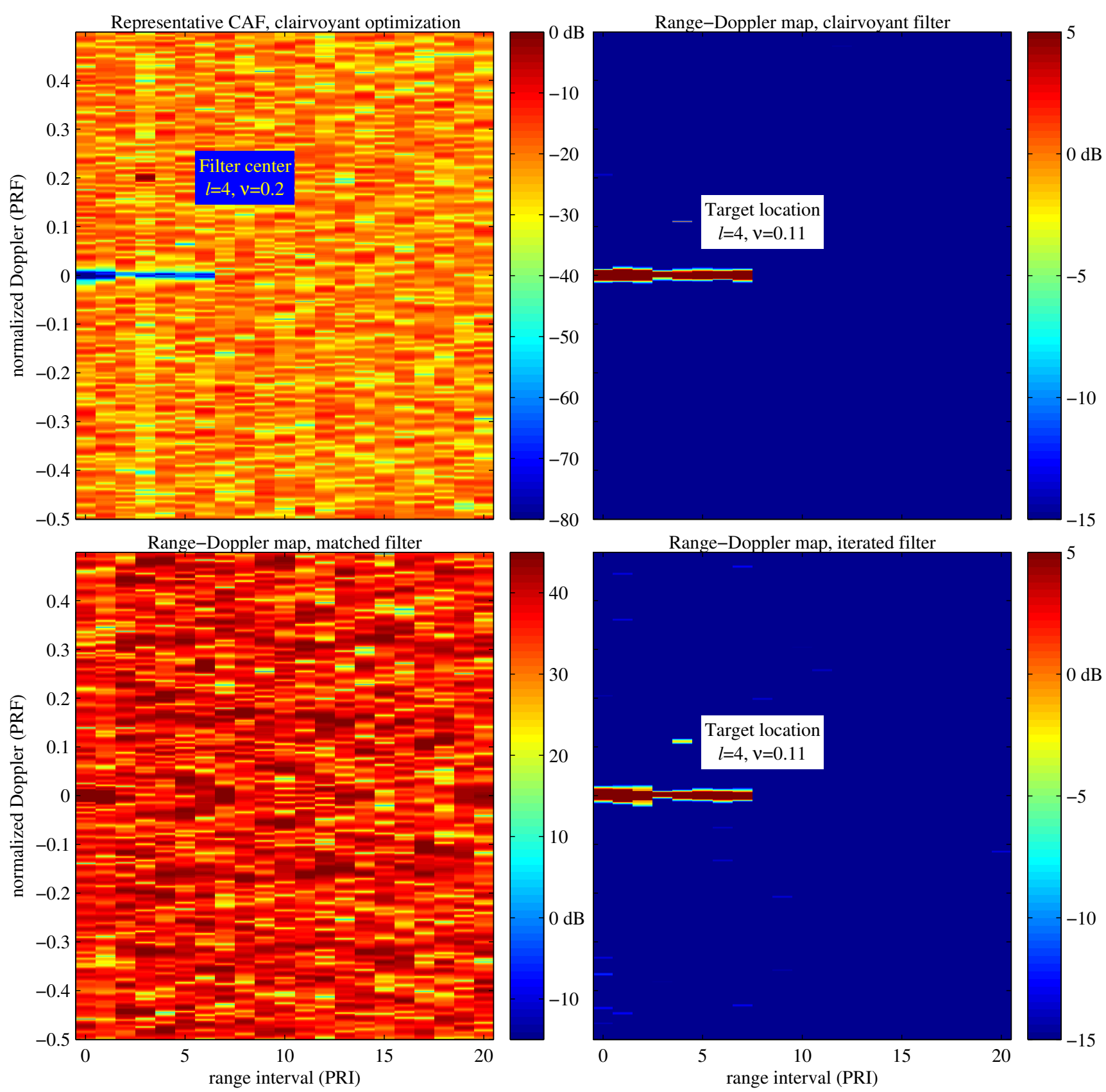

Fig. 3. Optimized cross-ambiguity function and range-Doppler maps for coherent-on-receive example. At top left is the CAF of a representative filter optimized with prior knowledge of the clutter distribution. The clutter notch and the peak at the filter center are clearly visible. At top right is the range-Doppler map resulting from the clairvoyant optimization, with the clutter ridge and single target clearly visible. At bottom left is the range-Doppler map resulting from matched filtering; here clutter obscures all cells. At bottom right is the map after 20 iterations, with the clutter ridge and target again visible.

\section{REFERENCES}

[1] D. K. Barton, Modern radar system analysis. Artech House, 1988.

[2] S. Carlsson, "MTI-filtering for multiple time around clutter suppression in coherent on receive radars," in Proc. of the Int'l Radar Conf., London, 1982.

[3] F. Lin and M. Steiner, "New techniques for radar coherent range ambiguity resolution," in Proc. of the IEEE Radar Conf., 2001, pp. 99-104.

[4] S. Blunt, A. Shackelford, K. Gerlach, and K. Smith, "Doppler compensation \& single pulse imaging using adaptive pulse compression," IEEE Trans. on Aerospace and Electronic Systems, vol. 45, no. 2, pp. 647-659, 2009.

[5] T. Higgins, S. Blunt, and A. Shackelford, "Time-Range adaptive process- ing for pulse agile radar," in Waveform Diversity and Design Conference (WDD), 2010 International, 2010, pp. 115-120.

[6] T. Higgins, K. Gerlach, A. K. Shackelford, and S. D. Blunt, "Aspects of non-identical multiple pulse compression," in IEEE Radar Conf., Kansas City, MO, May 2011.

[7] M. S. Lobo, L. Vandenberghe, S. Boyd, and H. Lebret, "Applications of second-order cone programming," Linear Algebra and its Applications, vol. 284, pp. 193-228, Nov. 1998.

[8] J. O. Coleman and D. P. Scholnik, "Design of nonlinear-phase FIR filters with second-order cone programming," in Proc. Midwest Symp. on Circuits and Systems, Las Cruces NM, Aug. 1999.

[9] J. O. Coleman, "Systematic mapping of quadratic constraints on embedded FIR filters to linear matrix inequalities," Princeton, NJ, Mar. 1998. 